\title{
The Evolution of Lepton Collider Detectors
}

\author{
John Hauptman \\ * \\ Iowa State University \\ E-mail: hauptman@iastate.edu
}

The character and capabilities of lepton storage ring detectors have evolved from the simple twoscintillators-in-coincidence to the very sophisticated, and future lepton collider detectors must be better by factors of 2 in calorimeter energy resolution and by factors of 10 in tracking momentum and impact parameter resolutions. I will discuss the configurations of recent and current detectors and potential future detectors at a linear electron collider (CLIC/ILC) or a Muon Collider.

8th International Conference on Nuclear Physics at Storage Rings-Stori11,

October 9-14, 2011

Laboratori Nazionali di Frascati, Italy

*This was a most enjoyable conference, superbly configured and delivered ("bellisima”, according to Victor Varentsov). I thank the organizers, Richard Wigmans and Paola Gianotti, for the invitation. 


\section{Introduction}

This paper is about the detectors, not the colliders or the physics, each of which have no purpose without the detectors. It is the detectors that hold the field of storage ring physics together. A graduate student, Sung Keun Park, put it most succinctly:

Now I see. The experimentalist connects the nut and bold to the Feynman diagram.

This simple statement expresses the exceptional reach of the experimental physicist, from the engineering details to the theoretical understandings, without which we would have learned nothing about Nature.

These detectors are driven by two terms: the scientific expectations (or "vision") and the available technologies. In the beginning with AdA, one could say that the expectations were "can we see collisions?", or with Adone "can we check QED?". By the 1970s after Ada, Adone, and CEA, the expectations were more sophisticated and, in the words of Burt Richter, circa 1971, were that the simple virtual photon created by the $e^{+} e^{-}$annihilation would "couple to everything".

Subsequent detectors were often built to study specific particles, e.g., the quarks $c, b$ and the lepton $\tau$ (Mark 3, BES), or the $Z^{0}$ (SLD, ALEPH, DELPHI, OPAL, L3). Some detectors were built to search for expected quarks, e.g., the top quark $t$ (TOPAZ, VENUS, Mark 2, HRS, MAC, TPC). Future lepton colliders are expected to study the Higgs boson $(H)$, SUSY, etc., after they are discovered and their masses roughly measured at the LHC.

The available technologies have dramatically expanded in scope and capability since AdA. I will only concentrate on tracking, calorimetry and the magnetic field, since these three largely define the detector. The so-called vertex chamber and the chambers for the so-called muon system are varied, but do not influence the main features of the detector.

For tracking, the first detector could have been two scintillators in coincidence, later replaced by spark chambers both optical and electronic, then MWPCs and drift chambers from Charpak's work. The advent of TPCs began in mid-1970s following drift chamber ideas and TPCs remain current today with a large TPC proposed for the International Large Detector (ILD) at the International Linear Collider (ILC). Silicon strip and pixel tracking devices are actively being developed and designed both as stand-alone tracking systems for the Silicon Detector (SiD) and in conjunction with a TPC (ILD).

For calorimetry, the first detector was very close to being two scintillators in coincidence with a sheet of $\mathrm{Pb}$ between them, and this "shower counter" mentality persisted for several years. Subsequent calorimeters have followed the "dagwood" design, Fig. 1, by stacking up many layers of absorber-sensor (e.g., $\mathrm{Pb}$-scintillator, $\mathrm{Pb}$-gas, $\mathrm{Pb}-\mathrm{LAr}$, etc.) motivated by the notion that more measurements must be better. Extreme cases of this are the calorimeters designed for "Particle Flow Analysis" (PFA) that seek to match incoming momentum-measured charged tracks with tracks and energy deposits within the calorimeter volume, necessitating cubic centimeter channel volumes and, therefore, $\sim 50 \times 10^{6}$ channels for a collider calorimeter. I will not discuss these calorimeters, but rather I will discuss and show data from "dual readout" calorimeters that I believe will dominate calorimetry in future lepton detectors.

Apart from the more-or-less obvious increase in the magnetic fields for charged particle momentum measurement $\left(\sigma_{p} / p \sim 1 / B\right)$, and the obvious improvements in energy resolutions of 
calorimeters (both electromagnetic and hadronic), the final evolution involves particle identification. At an early $e^{+} e^{-}$collider, simple $e^{ \pm}-\pi^{ \pm}$discrimination was important to distinguish QED events from "hadronic" events ( $e^{+} e^{-} \rightarrow q \bar{q} \rightarrow$ hadrons), and this was built into the early detector designs, e.g., barioni-antibarioni at Ada, and also in the Magnetic Detector several years later. It is interesting to recall that the discovery of the $\tau$ lepton with the Magnetic Detector was not easy or obvious, primarily because the shower counter of $\mathrm{Pb}$-scintillator layers was inefficient. ${ }^{1}$ The identification of $\tau$ events was so uncertain that in the early months not all members of the collaboration believed it was evidence for a new lepton.

Particle identification became of paramount importance in later detectors after the discovery of charm and its decay chain $c \rightarrow s \rightarrow K^{ \pm}$ to mesons, necessitating particle identification of $e^{ \pm}-\pi^{ \pm}-K^{ \pm}$. The subsequent discoveries of $b$ and $t$ quarks and the full decay chain $t \rightarrow$ $b \rightarrow c \rightarrow s \rightarrow u / d$ reinforced this need. The measurements of small branching ratios to high precision in the $c, b$-factory detectors BELLE, BaBar, and CLEO demanded ultra-high quality particle identification throughout the multi-GeV energy region on all charged particles $e^{ \pm}-$ $\mu^{ \pm}-\pi^{ \pm}-K^{ \pm}-p / \bar{p}$. Future lepton collider detectors must do more: they must measure every particle of the standard model, i.e., every particle in Fig. 6, including precision measurements of the $W^{ \pm}$and $Z^{0}$ bosons in their hadronic, or $q \bar{q}$, decay modes.

\section{Early Detectors}

The Livingston plot is famous for making obvious the spectac-

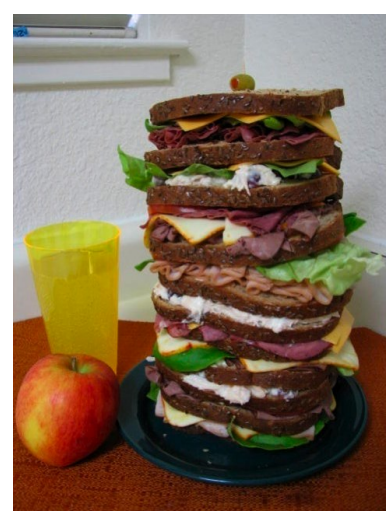

Figure 1: A "dagwood" calorimeter resembles a dagwood sandwich: both have many layers. ular exponential increases in laboratory particle energies from accelerators, but there is no equivalent plot for detectors. One can plot maximum B versus calendar year, or $\sigma_{E} / E$, or $\sigma_{p} / p$, or even the degree and efficacy of particle identification, versus calendar year [1], but this is only revealing as far as the available technologies are concerned. The second factor is the then-current vision of the physics, or the scientific goals. One might say that at the very first colliders CBX and AdA, the experimenters' goal was to see scintillation counters firing in coincidence. This would be enough, at first.

\subsection{Richter's Magnetic Detector}

A group at SLAC was designing a detector for the proposed, but not approved, collider called the Stanford Positron Electron Asymmetric Rings (SPEAR). Richter later wrote "While SPEAR was being designed, we were ... thinking about the [detector]. In the 1965 SPEAR proposal, we had described two different kinds of detectors: the first, a non-magnetic detector that would have looked only at particle multiplicities and angular distributions, with ... crude particle-identification ...; the second, a magnetic detector that could add accurate momentum measurement ..."

As we will see, almost all future collider detectors, including most in $p p$ and $p \bar{p}$ colliders, are of this same magnetic field design: a solenoid with Fe flux return.

\footnotetext{
${ }^{1} \mathrm{~A}$ over-eager technician had cleaned the scintillators with a cleaning compound that left micro-cracks on the scintillator surface, and the mean-free-path of light was very short.
} 


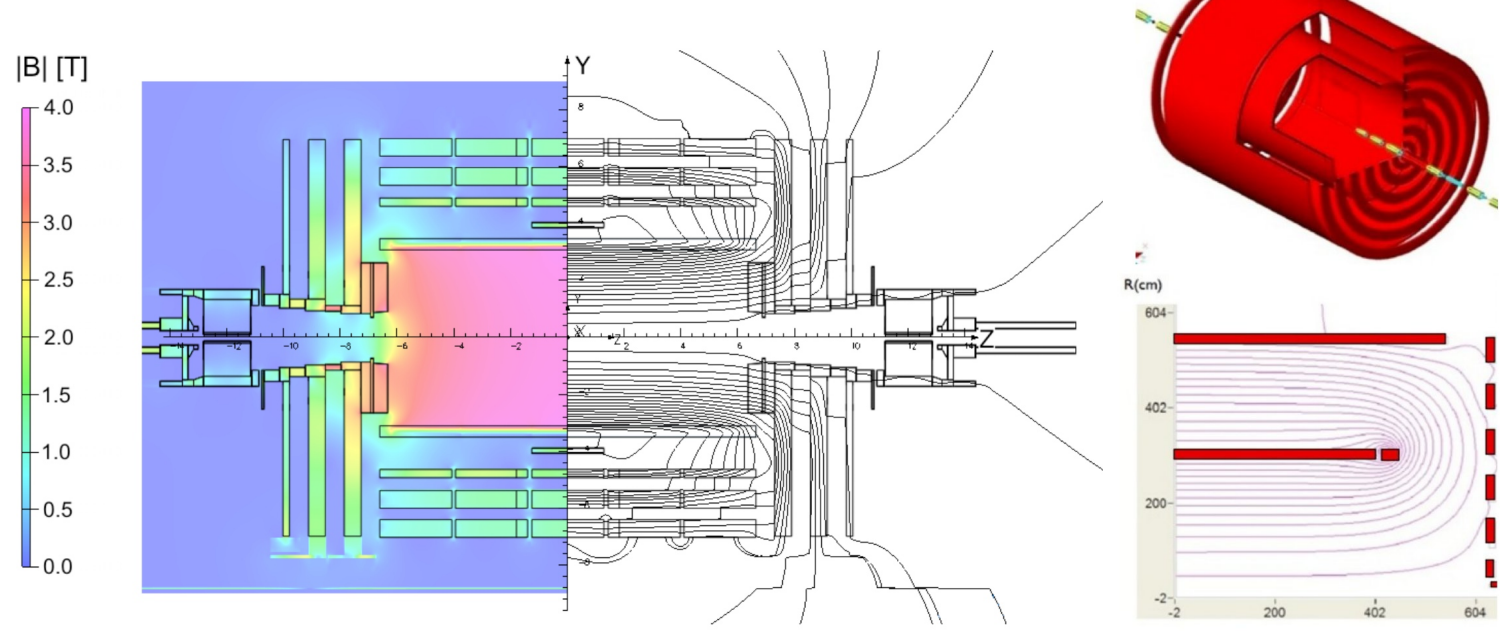

Figure 3: (a) The CMS field trying to minimize $U=(1 / 2 \mu) \int B^{2} d V$; and, (b) a dual-solenoid field, by A. Mikhailichenko.

\subsection{Steinberger's ALEPH}

Young physicists and students seldom, if ever, see the very beginning stages of the design of a detector, but one instructive example is the ALEPH detector built for the Large Electron Positron (LEP) collider. This group started to meet informally at CERN and, as Jack Steinberger wrote in his memoir [2], "We had open meetings about once a week ... at which all important design features ... were ..decided." These meetings would have been the best possible graduate seminar [4] . There were five main decisions:

1. "The magnetic field should be a superconducting solenoid with $1.5 \mathrm{~T}$... a technical challenge."

2. "The main tracking should be ... a TPC."

3. "The electromagnetic calorimeter should be optimized for spatial rather than energy resolution ... for particle identification."

4. "The hadronic calorimeter should use the iron return yoke."

5. "The detector naturally consists of a 'barrel' and two 'end caps'."

For both Richter and Steinberger, the detector design was done at a large laboratory with their concentrations of talent, not at a university. This highly creative process involved scientific and technical judgments, broad understanding of the precisions and purposes of detectors, the balance between detectors within a large experiment, and all with an eye on the costs and future problems of calibration and reliability. The ALEPH detector chose proven technologies, to be scaled up, in a balanced and coordinated detector. Let's take each in turn: magnetic field, tracking, calorimetry, and geometry.

\subsubsection{Magnetic field}

All lepton collider detectors now use a solenoidal magnetic field for tracking, following Richter's magnetic detector. As an example, the CMS field is shown in Fig. 3(a) with a substantial number of field lines going off to infinity (or the elevator shafts). The main problem is that $\mathrm{Fe}$ saturates at 
$1.8 \mathrm{~T}$ but the solenoid generates $4.0 \mathrm{~T}$. The magnetic configuration will minimize the total magnetic energy of the system, $U=\frac{1}{2 \mu} \int B^{2} d V$, and since the permeability of $\mathrm{Fe}, \mu$, is 1000 times the permeability $\mu_{0}$ of air, $\mathrm{U}$ is dramatically minimized only when the flux is inside $\mathrm{Fe}$. But, when the $\mathrm{Fe}$ is saturated, there is no energy advantage, and the flux goes off to find an unsaturated piece of $\mathrm{Fe}$ outside the detector.

In spite of its popularity, an Fe return is not necessary, and the 4th concept detector [3] has advocated a second outer solenoid, driven in the opposite direction with end coils, that has a zero fringe field, among many other scientific and technical advantages for detector without the iron sarcophagus :

1. the internal forces on the detector are minimal, and the two solenoid are self-stable;

2. the zero fringe field means no stray fields on the delicate final focus magnetic elements;

3. $15 \mathrm{kt}$ lower mass makes all mechanical problems in the IR easier;

4. the field can be reversed to cancel detector asymmetries; also, can

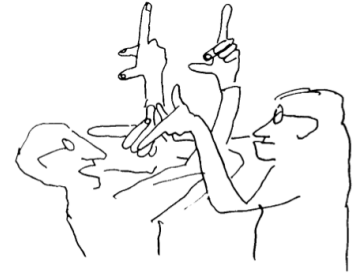

MAGNETIC PISCUSSION

Figure 2: A "magnetic discussion", by Bruno Touschek. run at any field, including zero;

5. the annulus between the solenoids is used to re-measure $\mu^{ \pm}$, and can be instrumented with novel devices, if desired; and,

6. the detector can easily be disassembled or re-configured since the calorimeter is the only item with any appreciable mass.

\subsubsection{Main tracking}

Future lepton colliders will require extreme momentum resolution, but also particle identification, and the chambers must present low-mass to the electromagnetic debris from the beam crossings. There are three designs for a future $\mathrm{TeV}$-scale collider:

1. an all-silicon, 5-layers, $5 \mu \mathrm{m}$ point precision $(\mathrm{SiD})$

2. a very high-performance TPC (ILD)

3. a KLOE-like drift chamber with cluster-timing and cluster-counting (4th)

These are dramatically different choices, and all yield about the same momentum resolution. It is often the case that tracking systems fail on backgrounds, not on physics or technology, and that will likely be the case with these three choices. I like the KLOE-

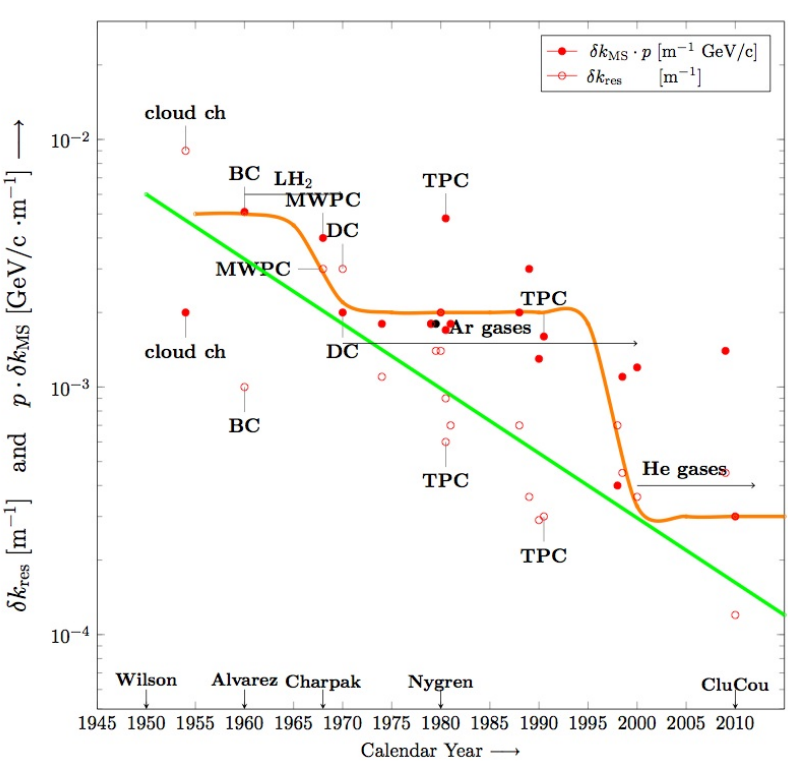

Figure 4: The evolution of the two curvature terms for a main tracker, plot by F. Grancagnolo.

like chamber because it is nearly mass- 

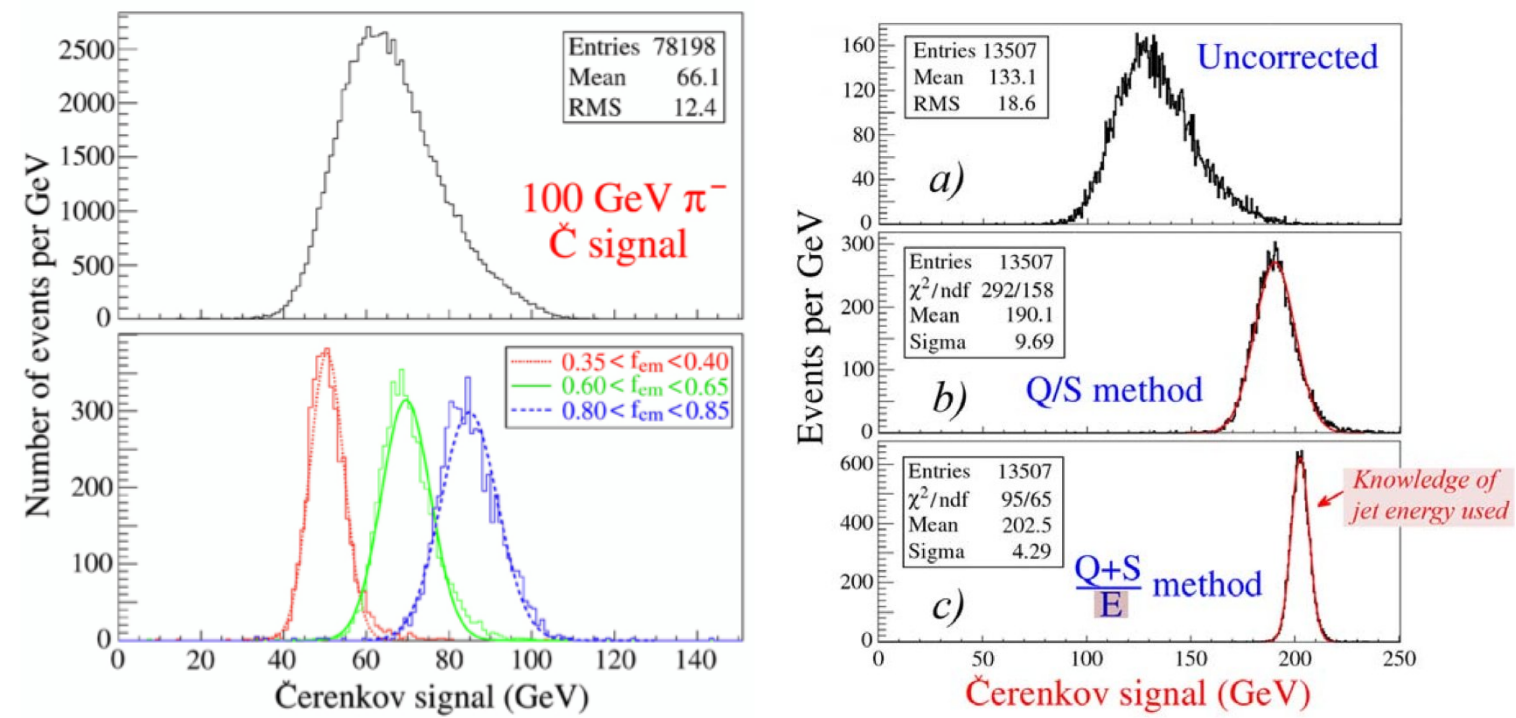

Figure 5: DREAM data (on left) shows the raw Čerenkov response to $100 \mathrm{GeV} \pi^{-}$beam particles and (below) the response functions for slices of the electromagnetic fraction, $f_{E M}$, of 0.35-0.40, 0.60-0.65, and $0.80-0.85$, each of which is narrow and Gaussian. On the right, (a) the raw response to multi-particle (or "jet") events at $200 \mathrm{GeV}$; (b) the response function when $f_{E M}$ is used to equalize the relative response; and, (c) the response when leakage fluctuations are suppressed.

less, intercepting far less debris from the IP, and it contributes to particle identification through $d N / d x$ Poisson cluster counting with roughly $3 \%$ resolution on specific ionization.

The two curvature uncertainty terms, $\delta k_{M S}$ for multiple scattering and $\delta k_{\mathrm{RES}}$ for sagitta resolution, are shown in Fig. 4 from cloud chambers and bubble chambers through to the ultra-low mass KLOE-like chambers. The curvature uncertainty due to point resolution has decreased uniformly for 60 years, whereas the uncertainty due to multiple scattering has fallen in steps as chambers have progressed from bubble and spark chambers, to drift chambers and TPCs with Argon gases, then to drift chambers with Helium gases.

\subsubsection{Calorimetry}

Electromagnetic calorimeters are easy to build and almost always perform well. The main challenge for a future detector is the hadronic calorimeter that must achieve jet 4-vector reconstruction with such high precision that $W^{ \pm} \rightarrow q \bar{q}$ and $Z^{0} \rightarrow q \bar{q}$ decays are measured with resolutions similar to electromagnetic resolutions on electrons and photons. Of the two main hadronic calorimeter technologies, PFA and dual-readout, I prefer dual-readout [5] because of its experimentally demonstrated energy resolution and particle identification capabilities in beam tests.

A dual-readout calorimeter measures the electromagnetic fraction each event, i.e., the relative $\pi^{0} \rightarrow \gamma \gamma$ and $\pi^{ \pm}$content, by embedding both scintillating $(S)$ fibers and clear $(C)$, or Čerenkov, fibers within the absorber mass. The $\mathrm{S}$ signal is proportional to total energy loss, whereas the $C$ signal is generated predominantly by the relativistic $e^{ \pm}$from the $\gamma$-initiated showers from $\pi^{0} \rightarrow \gamma \gamma$ decay. The ratio $C / S$ is a direct measure of the electromagnetic content, or EM fraction $f_{E M}$, and enables one to determine the response of the calorimeter. This is illustrated in the data from 
DREAM in Fig. 5 in which a selection on $f_{E M}$ results in a series of narrow Gaussians. Thus, the wide asymmetric hadronic response is actually a sum of narrow Gaussians. Summing these using the known $f_{E M}$ eliminates the several deleterious effects of the different instrumental responses to electromagnetic and hadronic energy losses and results in energy linearity with only calibration by electrons, a Gaussian response, and excellent energy resolution of approximately

$$
\frac{\sigma_{E}}{E} \approx \frac{30 \%}{\sqrt{E}} .
$$

The dual-readout collaboration, DREAM [5], has performed dual-readout in several crystals, in addition to measuring the invisible energy and the fluctuations in binding energy losses through measurements of the $\mathrm{MeV}$ neutron content of individual hadronic showers. Simulations indicate hadronic energy resolutions between $25 \% / \sqrt{E}$ and $30 \% / \sqrt{E}$ are easily possible with dual-readout techniques.

\section{Particle identification}

There are several powerful particle identification techniques [6] that are unique to dual-readout calorimeters which cannot be described here for wont of space. The necessary goal is to identify (with high probability and low contamination) all of the particles in Fig. 6.

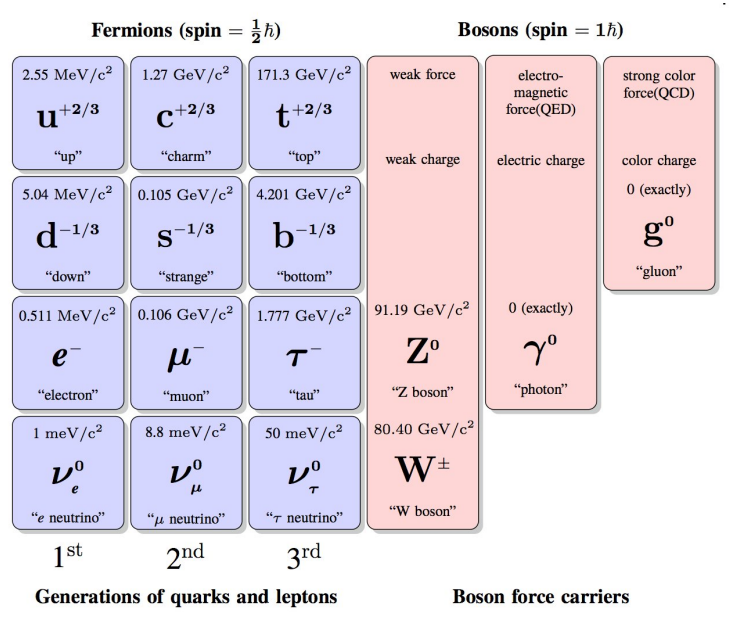

Figure 6: All particles of the standard model: the fermions are arranged in the usual way as generations. The bosons are matched vertically with the fermions which carry the corresponding coupling.
The light quarks $u, d$ and the gluon $g$ are measured as "jets" in the detectors and largely indistinguishable from each other event-by-event. Their energy resolution would be the dual-readout hadronic resolution of $\sigma_{E} / E \approx 30 \% / \sqrt{E}$.

The photon $\gamma$ and the electron $e^{ \pm}$are measured as electromagnetic in the dualreadout calorimeter $\left(\sigma_{E} / E \approx 10 \% / \sqrt{E}\right)$ and identified with the main tracker. In addition, the $e^{ \pm}$is distinguished from the more numerous $\pi^{ \pm}$by several unique measurements in the dual-readout calorimeters [6].

The strange $s$, charm $c$, and bottom $b$ quarks are measured in their decays to $K^{ \pm}$ which in turn are identified by specific ionization, or $d E / d x$ measurement. In addition, the $c$ and $b$ quarks are tagged by the finite lifetimes and $m m$-decay lengths of $D$ and $B$ mesons.

The $\tau^{ \pm}$lepton is identified both by its topologies (1-prong or 3-prong) and its sub-mm decay length. The $\mu^{ \pm}$is identified by its penetration of the calorimeters and its unique signature in a dual-readout calorimeter, $S-C \approx 1 \mathrm{GeV}$, while $(S+C) / 2$ is the total bremsstrahlung energy for a $\mu^{ \pm}$, or the energy of the hadron.

Measuring $W^{ \pm} \rightarrow q \bar{q}, Z^{0} \rightarrow q \bar{q}$ and $t \rightarrow W^{+} b W^{-} \bar{b}$ hadronic decays with high precision is shown in Fig. 7(a) for $W \mathrm{~s}$ and Zs produced in a simulated SUSY final state, and in Fig. 7(b) for top 

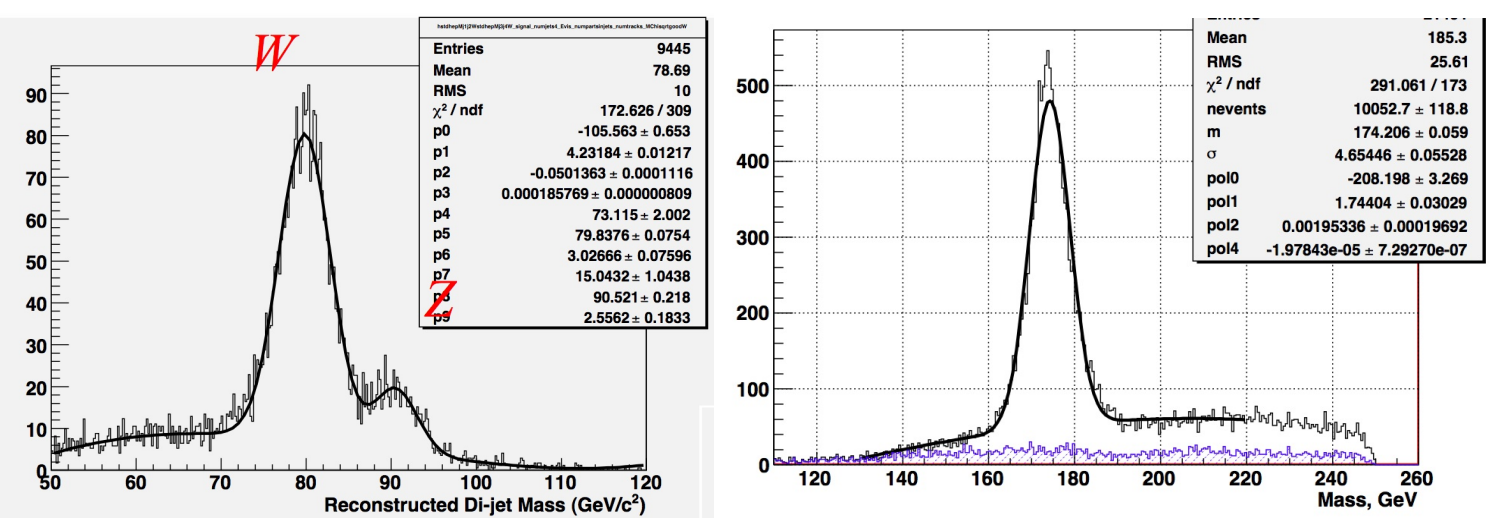

Figure 7: (a) The two-jet mass showing the di-jet mass resolution; (b) the top quark mass in the six-jet all-hadronic decay mode.

quark $t$ decays to all-jets. The di-jet mass resolution on the $W^{ \pm}$and $Z^{0}$ is about $\sigma_{M j j} \approx 3 \mathrm{GeV} / \mathrm{c}^{2}$, compared to the $W-Z$ mass difference of $10.8 \mathrm{GeV} / \mathrm{c}^{2}$.

Lastly, the $v$ 's are tagged by subtraction, i.e., their missing momentum vector is $\vec{p}_{v}=-\Sigma_{k} \vec{p}_{k}$. The good energy resolution on all the $\vec{p}_{k}$ yields a good estimate of the neutrino 3-vector.

\section{Summary}

We don't get to built TeV colliders very often and, depending on the fate of the LHC, we might have just one more chance to built one more world collider. I believe that the detectors at this collider must be near perfect in all respects.

\section{References}

[1] These plots are available in the figures of the talk itself, available at http://www.lnf.infn.it/conference/stori11/scientific_program.html.

[2] “Learning About Particles - 50 Privilieged Years," Jack Steinberger, Springer, 2004.

[3] "Letter of Intent from the fourth detector ("4th") collaboration at the international linear collider," Drobychev, G. et al. (2009), available at http://www.4thconcept.edu/4LoI.pdf.

[4] "Particle Physics Experiments at High Energy Colliders," John Hauptman, Wiley-VCH, 2011 (textbook).

[5] "Dual-Readout Calorimetry for High-Quality Energy Measurements," R. Wigmans, CERN-SPSC-2010-012, SPSC-M-771, 31 March 2010. Now, officially RD52.

[6] "Dual-Readout, particle identification, and 4th," V. Di Benedetto, J. Hauptman, F. Ignatov, and A. Mazzacane, Nucl. Instr. Meths. A (2010) doi:10.1016/j.nima.2010.02.207. 